\title{
Riscos e efeitos colaterais do uso de anorexígenos em mulheres no estado de São
}

\section{Paulo}

\author{
Risks and side effects of the use of anorectics in women in the state of São Paulo \\ Riesgos y efectos colaterales del uso de anorexígenos en mujeres del estado de São Paulo
}

Recebido: 23/09/2021 | Revisado: 30/09/2021 | Aceito: 03/10/2021 | Publicado: 04/10/2021

\author{
Thamires Maria de Macedo da Cunha \\ ORCID: https://orcid.org/0000-0002-1926-1361 \\ Universidade Anhembi Morumbi, Brasil \\ E-mail: tmmcunha@yahoo.com.br \\ Vitória Maria Branco Sestito \\ ORCID: https://orcid.org/0000-0003-0098-9552 \\ Universidade Anhembi Morumbi, Brasil \\ E-mail: vitoria.sestito@outlook.com \\ Isabella Oliveira Campanha \\ ORCID: https://orcid.org/0000-0001-5746-8884 \\ Universidade Anhembi Morumbi, Brasil \\ E-mail: campanhaisabella@outlook.com \\ Kerolaine Ladeira Moreira \\ ORCID: https://orcid.org/0000-0002-6763-8186 \\ Universidade Anhembi Morumbi, Brasil \\ E-mail: keromoreira089@gmail.com \\ Gustavo José Vasco Pereira \\ ORCID: https://orcid.org/0000-0001-9811-7220 \\ Universidade Anhembi Morumbi, Brasil \\ E-mail: gustavo.pereira@anhembi.br
}

\begin{abstract}
Resumo
A obesidade é uma patologia que atualmente apresenta um dos maiores problemas nutricionais podendo acarretar no desenvolvimento de diversas injurias à saúde. $\mathrm{O}$ tratamento desta consiste na mudança de hábitos diários e alimentares ou, em alguns casos, no uso de medicamentos anorexígenos. Esses medicamentos, além do tratamento da obesidade, também estão relacionados a fatores como a insatisfação corporal e dos padrões impostos pela sociedade sobre a aparência das mulheres. O estudo tem como objetivo avaliar e compreender os principais riscos e efeitos colaterais do uso dos anorexígenos em mulheres no estado de São Paulo. O método para o desenvolvimento do presente artigo deuse através de artigos científicos em plataformas como Scientific Electronic Library Online (SciELO), PubMed, Google Acadêmico e Science Direct, além da utilização de questionário online sendo divulgado pelas mídias sociais que obteve 217 respostas. Ao ser avaliada a utilização dos anorexígenos e suas condições de uso percebeu-se que o medicamento mais procurado foi a sibutramina com 41,94\%. Foi também observado uma baixa adesão da associação do tratamento farmacológico com atividades físicas e mudanças alimentícias. A maioria das mulheres entrevistadas apresentaram efeitos adversos, tais como náuseas, dor de cabeça, diarreia, mudanças de humor e boca seca. E 47,50\% das participantes não obtiveram acompanhamento profissional, o que demonstra a falta de conhecimento perante aos riscos dos anorexígenos, uma vez que 23,04\% veem como uma forma de emagrecimento rápido.
\end{abstract}

Palavras-chave: Anorexígenos; Tratamento; Obesidade; Mulheres; Risco.

\begin{abstract}
Obesity is a disease that is currently one of the biggest nutritional problems that can lead to the development of several health injuries. The treatment of the disease consists in the change of daily and eating habits or, in some cases, using anorectics. These medications, in addition to treating obesity, are also related to factors such as body dissatisfaction and the standards imposed by society about women's appearance. This study aims to evaluate and understand the main risks and side effects of the use of the anorectics in women in the state of São Paulo. The method for the development of the present article was through scientific articles on platforms such as Scientific Electronic Library Online (SciELO), PubMed, Academic Google and Science Direct, and the use of online questionnaire disclosed through social media, which obtained 217 responses. When evaluated the use of anorectics and their conditions of use, it was noticed that the most sought drug was sibutramine with $41,94 \%$. It was also observed that there was a low adherence to the pharmacological treatment associated to physical activity and dietary changes. Most interviewed women presented side effects such as nauseas, headache, diarrhoea, mood swings and dry mouth. And
\end{abstract}


$47,50 \%$ of the participants did not received professional monitoring, which demonstrates the lack of knowledge regarding the risks of anorectic drugs, since $23,04 \%$ see it as a way of quick weight loss.

Keywords: Anorectics; Treatment; Obesity; Women; Risk.

\begin{abstract}
Resumen
La obesidad es una patología que em la actualidad presenta uno de los mayores problemas nutricionales, que pueden conducir al desarollo de varias lesiones a la salud. El tratamiento de esta consiste en cambiar los hábitos alimeticos y diarios, en algunos casos, utilizar medicamento anorexígenos. Estas drogas, además de tratar la obesidade, también están relacionados con factores como la insatisfacción corporal, y los estándares impuestos por la sociedad sobre la apariencia de las mujeres. El estudio tiene como objetivo evaluar y comprender los principales riesgos y efectos colaterales del uso dos anorexígenos en mujeres del Estado de São Paulo. El método para el desarrollo del presente artículo fue a través de artículos científicos en plataformas como Scientific Eletronic Library Online (SciELO), PubMed, Google Acadêmico y Science Direct, además de utilización de un cuestionario online que se difunde en las redes sociales, el cual obtuvo 217 respuestas. Al evaluar el uso de anorexígenos y sus condiciones de uso, se notó que el fármaco más buscado fue la sibutramina con 41,94\%. También se observo uma baja adherencia en la asociación del tratamiento farmacológico con la actividad física y los cambios dietéticos. La mayoría de las mujeres entrevistadas presentaron efectos adversos como náuseas, dolor de cabeza, diarrea, cambios de humor y boca seca. Y el 47,50\% de las participantes no recibió seguimiento profesional, lo que demuestra el desconocimiento sobre los riesgos de los anorexígenos, ya que el 23,04\% lo ve como una forma de pérdida de peso rápida.
\end{abstract}

Palabras clave: Anorexígenos; Tratamiento; Obesidad; Mujeres; Riesgo.

\title{
1. Introdução
}

A obesidade é uma doença crônica e multifatorial caracterizada pelo acúmulo excessivo de tecido adiposo no corpo. Esse tecido abundante é medido através do Índice de Massa Corporal (IMC), e o diagnóstico de obesidade é condicionado quando os valores se apresentam acima de $30 \mathrm{~kg} / \mathrm{m}^{2}$ (Pinheiro, Freitas \& Corso, 2004). Essa doença, além de prejudicar a saúde, pode levar ao aparecimento de demais patologias, como doenças cardiovasculares, diabetes, problemas respiratórios, hipertensão, podendo até mesmo proporcionar algum tipo de câncer. Ademais, a obesidade em mulheres também pode acarretar distúrbios reprodutivos (Ades \& Kerbany, 2002).

O tratamento caracteriza-se em múltiplas abordagens provenientes de uma equipe multidisciplinar, podendo inclusive implementar o uso de fármacos antiobesidade. Porém em alguns casos, os resultados seguem sendo insatisfatórios, o que pode levar o paciente a recuperar sua massa corpórea numa média de 2 anos. Os múltiplos profissionais de saúde envolvidos nesse tratamento devem se preocupar com a saúde física, social e psíquica; pois todos esses fatores são determinantes para um cuidado de excelência ao paciente obeso (Tavares, Nunes \& Santos, 2010).

O objetivo do tratamento através da mudança no estilo de vida é identificar e modificar os hábitos que contribuem para o peso do paciente; como por exemplo, alimentar-se em razão de fatores psicológicos (autoestima, depressão, ansiedade, transtornos compulsivos obsessivos, entre outros). Além de verificar se os fatores contribuintes para determinado peso estão atrelados à motivos que não sejam somente comportamentais, assim como fatores genéticos, metabólicos e hormonais. Essas mudanças na forma de tratamento precisam ser orientadas, com objetivos específicos, e com pequenas mudanças comportamentais (Wadden \& Foster, 2000).

Por se tratar de um problema de saúde complexo, é necessário o acompanhamento profissional do médico, nutricionista, psicólogo e farmacêutico (Mendes, 2018). Dietas popularmente mencionadas como “mágicas” possuem resultados duvidosos e podem acarretar consequências de curto, médio e longo prazo. Diferente das dietas prescritas por nutricionistas, que por se tratar de uma atividade privativa deste profissional, possuem embasamento científico para tal (Ades \& Kerbany, 2002).

A iniciação da terapia farmacológica é indicada somente caso haja alguma doença relacionada ao peso e aos demais tratamentos que vieram a ser ineficazes (Moreira \& Alves, 2015). A obesidade se tornou uma questão de saúde pública, e junto 
a automedicação, justamente por trazer o aspecto físico que o usuário pleiteia para si, sem haver a preocupação com os demais efeitos adversos acarretados pela classe medicamentosa (Campos, Oliveira, Silva \& Paiva, 2014).

Sendo assim, o tratamento da obesidade através de medicamentos está em constante mudança, pois tem sido bastante criticada nos últimos anos com a prescrição de medicamentos indevida e, a ausência pela procura de orientações relacionadas a mudanças de hábitos, como alimentação saudável, através de um planejamento alimentar e a prática de atividade física. O medicamento deve ser apenas um elemento adjuvante nesse conjunto de mudanças, visto que após a interrupção da administração é esperado que haja um aumento de massa corpórea em pacientes que apresentam esse perfil (Naccarato \& Lago, 2014).

Além da obesidade, outro conceito para o uso irracional dos anorexígenos advém de a "cultura da magreza" ser sinônimo de beleza física. O que fez com que o Brasil e muitos outros países, iniciassem uma espécie de epidemia através do uso irracional de medicamentos inibidores de apetite. Em especial no Brasil, a problemática é ainda maior, pois com os médicos realizando a individualização do tratamento, através de receitas para formulações manipuladas, é possível prescrever os anorexígenos associados de demais medicações, tais como hormônios e benzodiazepínicos (Nappo, Tabach, Noto, Galduróz \& Carlini, 2002).

A insatisfação corporal também acomete adolescentes de ambos os sexos, de modo que a maneira que percebem seu corpo, traz consequências negativas para sua saúde, por desejarem algo fora de seu estado nutricional. Este tipo de insatisfação pode ser visto como um marcador de risco para distúrbios alimentares (Dumith et al., 2012). Uma vez que a saúde é um estado de plenitude do indivíduo, e isso inclui uma mente saudável (Tavares et al., 2010).

A elaboração deste artigo possui como intuito avaliar e compreender os principais riscos, bem como efeitos adversos dos medicamentos anorexígenos de origem alopática em mulheres no estado de São Paulo. Além de realizar pesquisa de campo através das mídias digitais para coletar dados de maneira anônima para o desenvolvimento deste artigo, e dessa forma ser mais assertivo ao alertar o público-alvo sobre os principais riscos do uso deste grupo de medicamentos.

E assim conscientizar as pessoas, não somente mulheres, sobre a importância do acompanhamento profissional ao utilizar medicamentos emagrecedores.

\section{Metodologia}

O método utilizado para o desenvolvimento deste artigo original foi através de artigos científicos e livros relacionados ao assunto, com palavras-chave para busca, como: anfetaminas, obesidade, tratamento para obesidade, anorexígenos, emagrecedores; nas plataformas SciELO, PubMed, Google Acadêmico e Science Direct, a fim de revisar o tema escolhido para o desenvolvimento do artigo em questão entre os anos de 2000 e 2021. Este período foi selecionado devido aos estudos realizados sobre os fármacos anorexígenos e a evolução destes medicamentos, e do crescente número de casos de obesidade.

Este método foi realizado com o auxílio das literaturas como elaborar projetos de pesquisa e Metodologia do trabalho científico, como forma de embasamento nesta pesquisa de campo quantitativa (Gil, 2017; Marconi \& Lakatos, 2017)

Como parte do desenvolvimento, foi elaborado um questionário com 11 questões, conforme o Quadro 1, via formulário online onde apenas mulheres a partir de 18 anos poderiam responder para o levantamento de dados. Este formulário foi realizado via Google Forms e divulgado pelas mídias sociais no período de 23 de agosto de 2021 e 1 de setembro de 2021 , após a aprovação do Comitê de Ética de número 46982221.0.0000.5492 de parecer de número 4.925.901, onde 217 mulheres responderam. Este número de participantes foi definido devido as restrições para responder a pesquisa, e assim concluir e comparar com demais estudos com veracidade, através de números, os riscos e efeitos colaterais causados pelo uso de medicamentos emagrecedores em mulheres que residem no estado de São Paulo. 
Quadro 1. Questões

\begin{tabular}{|rl|}
\hline 1. & Quantos anos você tem? \\
\hline 2. & Qual medicamento para emagrecer você utilizou? \\
\hline 3. & Você conhece alguém, sem ser você, que já fez uso? \\
\hline 4. & Em poucas palavras, qual foi o motivo que te levou a utilizar esses medicamentos? \\
\hline 5. & Durante o uso, você teve acompanhamento com algum profissional da saúde? \\
\hline 6. & Caso a resposta anterior seja negativa, quem lhe passou o medicamento? \\
\hline 7. & Você apresentou resultados utilizando este medicamento? Em caso de a resposta ser \\
& positiva, este resultado continuou mesmo após a interrupção do uso? \\
\hline 8. & Você apresentou algum efeito colateral durante o tratamento? (Exemplos: náuseas, dor \\
\hline 9. & de cabeça, boca seca) \\
\hline 10. & Você realizou alguma atividade física junto com o uso desse medicamento? \\
\hline 11. & Você realizou alguma mudança em sua alimentação durante o uso do medicamento? \\
\hline
\end{tabular}

Fonte: Autores.

\section{Revisão de Literatura}

\subsection{Obesidade}

Após a Segunda Guerra Mundial os hábitos alimentares no hemisfério norte mudaram bem como a prática de atividade física. Isso acarretou o crescimento da obesidade, que é causado principalmente pela mudança no estilo de vida após 1945; onde a indústria estava cada vez mais potencializada e o capitalismo cada vez mais presente no urbanismo com a produção de bens duráveis. Esta mudança se alastrou para o hemisfério sul mais tardiamente, como no Brasil, que passou pelo processo de industrialização de bens duráveis nas décadas de 70 e 90, onde houve o grande crescimento da doença (Mendonça \& Anjos, 2004).

De acordo com a Organização Mundial da Saúde (OMS), a obesidade é um tema de saúde pública por prevalecer em todas as camadas da sociedade, incluindo crianças, adolescentes e adultos. O impacto da doença sobre o indivíduo apresenta-se pela influência na qualidade de vida, recursos de saúde, limitações de atividades físicas diárias, depressão e isolamento (Amaral \& Pereira, 2016). No Brasil, o maior índice de obesidade está entre as mulheres (Ferreira \& Benicio, 2015) uma vez que está associado com a distribuição e a morfologia do tecido adiposo resultando em alterações circulatórias e hormonais (Amaral \& Pereira, 2016).

A obesidade feminina tem sido apontada como um dos maiores problemas nutricionais da atualidade, devido as grandes mudanças sociais e econômicas que resultaram em modificações no estilo de vida, como as responsabilidades do mercado de trabalho, o que inclui a alimentação caracterizada pelo aumento de consumo de alimentos processados, ricos em gordura, açúcar e sal. Esses maus hábitos alimentares e a diminuição do gasto energético pelo sedentarismo, são fatores favorecedores do quadro da obesidade (Franke et al., 2010). Entretanto, a alimentação saudável encontra-se na escolha de alimentos, como a substituição da farinha branca e a inserção de fibras que são absorvidas mais lentamente, o que resulta em maior poder da saciedade como é o caso da farinha do maracujá amarelo (Balthar, Maciel \& Ferreira, 2021).

A obesidade também está associada à piora dos aspectos emocionais, dificuldades de integração social e baixa autoestima (Orsi et al., 2008). Isso está atrelado com a influência que a mídia causa na vida das pessoas e com os padrões 
impostos pela sociedade. Fazendo com que as mulheres comparem corpos e aparências com mulheres de revistas, propagandas, ou influenciadoras, gerando frustrações e em alguns casos até propiciando a depressão (Claudino \& Zanella, 2004).

Com a insatisfação das mulheres com relação ao seu corpo, uma vez que o padrão de beleza é o corpo esguio e fora dos padrões nutricionais (Ferrari \& Ferrari, 2017) são realizadas ações arriscadas e radicais, como prática de dietas restritivas afim de obter o corpo ideal (Kaoutar, Hilali \& Loukid, 2019) e jejum intermitente, sem orientações de um nutricionista; consumo de medicamentos como diuréticos e laxantes (sem prescrição ou necessidade), e até indução de vômitos (Barros, 2019); sendo atitudes que podem acarretar, manter ou renovar distúrbios alimentares (Kaoutar et al., 2019).

\subsection{História das Anfetaminas}

A primeira anfetamina foi sintetizada pelo químico alemão Lazar Edeleanu (Muakad, 2013) em 1887 na Alemanha, onde foi nomeado como 1-metil-2-feniletilamina (Marcon, Silva, Moraes, Martins \& Carpes, 2012) e, somente 40 anos depois foi inserido seu uso medicinal em tratamentos respiratórios, além de ser usado como estimulante do Sistema Nervoso Central (SNC) (Muakad, 2013). Na década de 30, a finalidade dessa substância era o tratamento de déficit de atenção e hiperatividade (Marcon et al., 2012).

A anfetamina recebeu o nome comercial de Benzedrina, onde tinha o intuito de combater a obesidade, a depressão e a congestão nasal (Sousa, 2015). O uso como estimulante do SNC foi bastante explorado durante a Segunda Guerra Mundial e a Guerra da Coreia, sendo recebida como suprimento, visto que durante a Segunda Guerra Mundial, notou-se que os efeitos colaterais da anfetamina poderiam trazer muitos benefícios para os soldados em batalha, já que sua ação farmacológica era de grande interesse por seu efeito estimulante, inibição de sono, e pela proporção de horas de energia; o que logo se tornou um grande aliado dos soldados, por mantê-los sempre acordados, além de inibir seu apetite (Muakad, 2013).

A anfetamina foi utilizada por Hitler em seu exército, ditando um ritmo mais "poderoso" ao Terceiro Reich. O uso de drogas beneficiou os soldados em combate, que obtiveram resultados satisfatórios para o exército nazista, fazendo com que houvesse mais pesquisas no mundo químico-farmacêutico, com a finalidade de potencializar as tropas (Soares, 2019). Com passar dos anos, o consumo da anfetamina aumentou de forma abusiva e descontrolada pelos tratamentos de perda de peso sem resultados positivos, o que gerava anseio recorrente da substância pelos usuários (Nateghi \& Effatpanah, 2020), uma vez que as anfetaminas geram dependência física e psicológica. Os jovens passaram a utilizá-las para atingirem os padrões estéticos da sociedade, seja corporal, emocional ou social (Moreira \& Alves, 2015), sendo o "ecstasy"; que é um derivado de anfetamina (Utrilla, 2000); uma forma de auxílio para atingir esses objetivos, pois faz com que os usuários se sintam importantes e desinibidos; o que significa que este jovem está "incluso" na sociedade (Moreira \& Alves, 2015).

\subsection{Anfetaminas: Mecanismo de Ação e Efeitos Adversos}

As anfetaminas são uma classe de medicamentos comumente conhecidas por causarem dependência e tolerância (Robledo, 2008). Com a individualização de tratamentos através de receitas para formulações manipuladas, há possibilidade da prescrição de anorexígenos associados de demais medicações, tais como hormônios e benzodiazepínicos (Nappo et al., 2002).

Os benzodiazepínicos são uma classe medicamentosa de ansiolíticos e hipnóticos, utilizados como sedativos e são moderadamente seguros quando usados a curto prazo. Em um período superior há 1 mês, aponta-se que metade dos pacientes desenvolveram dependência (Soyka, 2017). Em contraste, as anfetaminas possuem o uso recomendado de no máximo 3 meses e, ao final do tratamento, é realizado o sistema de desmame (Robledo, 2008).

Os benzodiazepínicos prescritos associados as anfetaminas em formulações magistrais são uma tentativa de equilibrar os efeitos da irritabilidade, ansiedade e dificuldade de dormir das anfetaminas, entretanto gera uma dependência de ambos os princípios ativos (Muakad, 2013). 
Além da dependência, as anfetaminas causam tolerância com seu uso abusivo, e exigem do organismo maiores doses para atingirem o efeito desejado, por ocorrer uma fosforilação do receptor que implica na separação do receptor acoplado a proteína G. Isso acontece tanto na tolerância crônica quanto na aguda, porém na aguda há a taquifilaxia, podendo levar o paciente a overdose (Robledo, 2008)

A estrutura química das anfetaminas, vem sendo modificadas para o âmbito terapêutico, ao longo dos anos, para que predomine o efeito anorexígeno no SNC. O femproporex e o clobenzorex (Figura 1) são exemplos disso, pois em suas estruturas há a substituição do anel benzênico por um grupamento amino volumoso. Estas mudanças auxiliam ao paciente em tratamento obterem maior efeito anorexígeno e menor capacidade de dependência (Utrilla, 2000).

Figura 1. Estrutura química do femproporex (A) e clobenzorex (B)

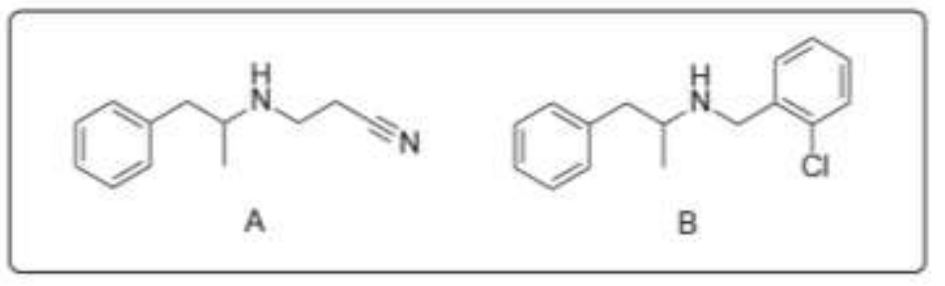

Fonte: Autores.

Nos Quadros 2 e 3 abaixo, é possível observar os principais medicamentos para emagrecer. No Quadro 2 encontramse os fármacos pertencentes a classe dos anorexígenos propriamente ditos, e no Quadro 3 os antidepressivos que devido ao seu mecanismo de ação inibem a fome. Em ambas os Quadros há a substância, seus mecanismos de ação, efeitos colaterais e doses diárias.

Quadro 2. Medicamentos anorexígenos

\begin{tabular}{|c|c|c|c|c|c|}
\hline Classe & Substância & Mecanismo De Ação & Efeito Colateral & Dose & Referência \\
\hline Catecolaminérgicos & Anfepramona & $\begin{array}{l}\text { Mecanismo de ação central. Inibe a } \\
\text { receptação de noradrenalina e } \\
\text { aumenta a interação desse } \\
\text { neurotransmissor com receptores } \\
\text { pós-sinápticos no hipotálamo, } \\
\text { trazendo sensação de saciedade e } \\
\text { diminuindo a fome. }\end{array}$ & $\begin{array}{l}\text { Taquicardia, náusea, boca seca, } \\
\text { vomito, constipação intestinal, } \\
\text { redução da libido, impotência sexual, } \\
\text { dor de cabeça, alucinação, } \\
\text { nervosismo, inquietação, insônia e } \\
\begin{array}{l}\text { depressão se houver intoxicação } \\
\text { aguda. }\end{array}\end{array}$ & $\begin{array}{c}40- \\
120 \mathrm{mg} / \mathrm{dia}\end{array}$ & $\begin{array}{l}\text { Duarte et al., } \\
2020\end{array}$ \\
\hline Catecolaminérgicos & Femproporex & $\begin{array}{l}\text { Ação noroadrenérgica, } \\
\text { dopaminérgica e nas vesículas pré- } \\
\text { sinápticas no centro do hipotálamo } \\
\text { agindo como um supressor de } \\
\text { apetite. }\end{array}$ & $\begin{array}{l}\text { Taquicardia, palpitação, hipertensão, } \\
\text { boca seca, desconforto abdominal, } \\
\text { vomito, diarreia, convulsões, } \\
\text { episódios psicóticos, depressão, visão } \\
\text { turva, irritabilidade, alterações } \\
\text { comportamentais e cardiovasculares, } \\
\text { alopecia e arritmia, que pode até levar } \\
\text { ao colapso cardiovascular. }\end{array}$ & $20-50 \mathrm{mg} / \mathrm{dia}$ & $\begin{array}{c}\text { Duarte et al., } \\
2020\end{array}$ \\
\hline Catecolaminérgicos & Manzidol & $\begin{array}{l}\text { Bloqueia a receptação da } \\
\text { noradrenalina e dopamina alterando } \\
\text { o mecanismo energético periférico e } \\
\text { aumentando a absorção de glicose } \\
\text { no musculo esquelético, } \\
\text { aumentando a atividade locomotora. }\end{array}$ & $\begin{array}{l}\text { Insônia, dor de cabeça, boca seca, } \\
\text { náusea, arrepios, irritabilidade, } \\
\text { fraqueza, palpitações, desconforto } \\
\text { gástrico, constipação, tontura, } \\
\text { vertigem e hiperidrose. }\end{array}$ & $1-3 \mathrm{mg} / \mathrm{dia}$ & $\begin{array}{c}\text { Duarte et al., } \\
2020\end{array}$ \\
\hline
\end{tabular}




\begin{tabular}{|c|c|c|c|c|c|}
\hline Serotoninérgicos & Sibutramina & $\begin{array}{l}\text { Inibe a captura de norepinefrina e } \\
\text { serotonina nos centros de } \\
\text { alimentação e saciedade no } \\
\text { hipotálamo, regulando a ingestão de } \\
\text { alimentos, aumentando a saciedade. }\end{array}$ & $\begin{array}{l}\text { Dor de cabeça, boca seca, náusea, } \\
\text { sudorese, dispneia, constipação } \\
\text { intestinal, vertigem, dor nas costas, } \\
\text { anorexia, alteração no paladar, } \\
\text { parestesia, dismenorreia, aumento da } \\
\text { frequência cardíaca, hipertensão } \\
\text { arterial, pressão arterial sistólica e } \\
\text { diastólica de repouso. }\end{array}$ & $10-20 \mathrm{mg} / \mathrm{dia}$ & $\begin{array}{l}\text { Fortes, } \\
\text { Guimarães, } \\
\text { Haack, Torres } \\
\text { \& Carvalho, } \\
\text { 2006; Rang \& } \\
\text { Dale, 2016 }\end{array}$ \\
\hline Inibidor de lipases & Orlistate & $\begin{array}{l}\text { Reduz a absorção de gordura, } \\
\text { inibindo a hidrolise dos } \\
\text { triglicerídeos sendo liberada nas } \\
\text { fezes. Seu mecanismo de ação inibe } \\
\text { de forma reversível e seletiva as } \\
\text { lipases gástricas e pancreáticas. }\end{array}$ & 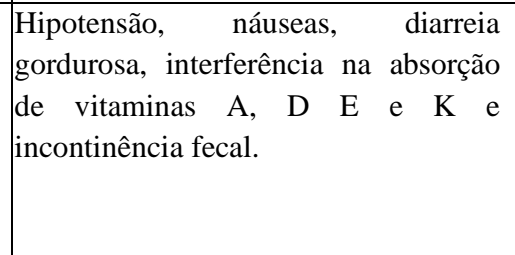 & $\begin{array}{c}\text { Max: } \\
\text { 120mg/dia }\end{array}$ & $\begin{array}{c}\text { Rang \& Dale, } \\
2016\end{array}$ \\
\hline Incretinomiméticos & Liraglutida & $\begin{array}{l}\text { Age nos receptores do cérebro } \\
\text { através da liraglutida, um } \\
\text { antagonista do receptor GLP-1, } \\
\text { ligando e ativando o receptor GLP- } \\
\text { IR, regulariza ndo o apetite, } \\
\text { reduzindo a ingestão de alimentos e } \\
\text { reduzindo também o peso corporal. }\end{array}$ & $\begin{array}{l}\text { Náusea, vomito, diarreia, constipação, } \\
\text { boca seca, dispepsia, gastrite, refluxo, } \\
\text { distensão abdominal, pancreatite, } \\
\text { hipoglicemia, desidratação, fadiga, } \\
\text { mal-estar, tontura, urticaria, } \\
\text { taquicardia, insuficiência renal aguda, } \\
\text { reação anafilática, insônia e aumento a } \\
\text { lipase e amilase. }\end{array}$ & $0,6-3 \mathrm{mg} /$ dia & $\begin{array}{l}\text { Skrsypcsak \& } \\
\text { Locatelli, } \\
\text { 2013; Conte } \\
\text { \& De } \\
\text { Campos, } 2015\end{array}$ \\
\hline
\end{tabular}

Fonte: Autores.

Quadro 3. Medicamentos antidepressivos utilizados para o emagrecimento

\begin{tabular}{|c|c|c|c|c|c|}
\hline Classe & Substância & Mecanismo De Ação & Efeito Colateral & Dose & Referência \\
\hline Serotoninérgicos & Fluoxetina & $\begin{array}{l}\text { Age } \\
\text { seletivamente a captura } \\
\text { da serotonina (5-HT), } \\
\text { reduzindo a fome e } \\
\text { aumentando a saciedade. }\end{array}$ & $\begin{array}{l}\text { Náusea, vomito, sonolência, dor de } \\
\text { cabeça insônia, ansiedade, redução } \\
\text { da libido, impotência sexual, retardo } \\
\text { na ejaculação, irritabilidade, } \\
\text { agitação, nervosismo e tremores, } \\
\text { além de inibir o metabolismo de } \\
\text { outros fármacos e dessa forma, corre } \\
\text { o risco de interação medicamentosa. }\end{array}$ & $\begin{array}{l}\text { 20-40mg/dia } \\
\text { Max: } \\
\text { 80mg/dia }\end{array}$ & $\begin{array}{l}\text { Santos, Silva \& } \\
\text { Modesto, } 2019 ; \\
\text { Rang \& Dale, } 2016\end{array}$ \\
\hline Catecolaminérgicos & Bupropiona & $\begin{array}{l}\text { Age inibindo a captura } \\
\text { de } \\
\text { reduzindo a compulsão } \\
\text { alimentar. }\end{array}$ & $\begin{array}{l}\text { Dor de cabeça, boca seca, agitação e } \\
\text { insônia, em grandes doses, pode } \\
\text { causar crises convulsivas. }\end{array}$ & $\begin{array}{l}150- \\
300 \mathrm{mg} / \mathrm{dia}\end{array}$ & Rang \& Dale, 2016 \\
\hline
\end{tabular}

Fonte: Autores.

\section{Resultados}

De acordo com a análise dos resultados da pesquisa "Riscos e Efeitos Colaterais do Uso de Anorexígenos em Mulheres no Estado de São Paulo”, o qual obteve-se 217 respostas é possível demonstrar os resultados dissertados abaixo. No Gráfico 1 visualiza-se em forma de porcentagem a faixa etária das participantes da pesquisa, sendo as mulheres entre 18 e 24 anos as que mais fazem o uso de anorexígenos.

Gráfico 1. Idade das mulheres entrevistadas 


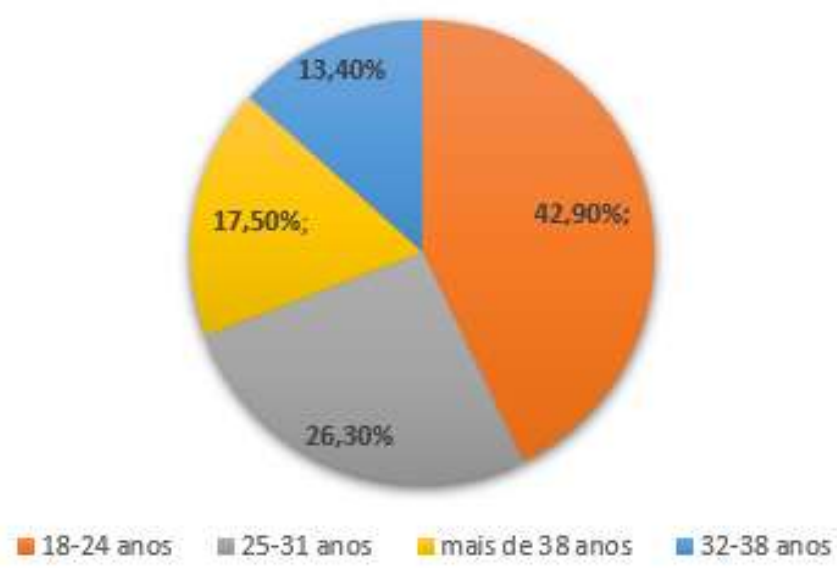

Fonte: Autores.

A relação dos medicamentos utilizados pelas entrevistadas é possível observar no Quadro 4 juntamente com porcentagens correspondentes. Para os antidepressivos, estão compreendidos em compilado a fluoxetina, bupropiona, sertralina e duloxetina. Além disso, 22,58\% das mulheres que responderam à pesquisa fizeram uso de mais de um medicamento anorexígeno.

Quadro 4. Medicamentos utilizados pelas entrevistadas

\begin{tabular}{|c|c|}
\hline Medicamentos & $\%$ \\
\hline Sibutramina & $41,94 \%$ \\
\hline Fitoterápicos & $17,51 \%$ \\
\hline Antidepressivos & $14,29 \%$ \\
\hline Orlistate & $11,06 \%$ \\
\hline Liraglutida & $10,59 \%$ \\
\hline Anfepramona & $8,29 \%$ \\
\hline Femproporex & $5,99 \%$ \\
\hline Topiramato & $5,06 \%$ \\
\hline Lisdexanfetamina & $1,84 \%$ \\
\hline Semaglutida & $1,38 \%$ \\
\hline Naltrexona & $0,46 \%$ \\
\hline Lorcarserina & $0,46 \%$ \\
\hline
\end{tabular}

Fonte: Autores. 
Quando questionadas em relação aos efeitos colaterais, 72,25\% das mulheres afirmaram que apresentaram, sendo possível que elas pudessem citar mais de um sintoma nessa questão. Os resultados desses efeitos podem ser observados no Quadro 5.

Quadro 5. Efeitos Colaterais apresentados pelas entrevistadas

\begin{tabular}{|c|c|}
\hline Efeito Colateral & $\%$ \\
\hline Náuseas e Enjoo & $36,80 \%$ \\
\hline Dor de cabeça & $29,70 \%$ \\
\hline Diarreia & $23,90 \%$ \\
\hline Mudanças de humor & $20 \%$ \\
\hline Boca seca & $20 \%$ \\
\hline Insônia & $10,90 \%$ \\
\hline Ansiedade & $7,10 \%$ \\
\hline Mal-estar & $5,20 \%$ \\
\hline Mudança da pressão arterial & $3,90 \%$ \\
\hline Taquicardia & $3,20 \%$ \\
\hline Prisão de ventre & $1,90 \%$ \\
\hline Sudorese & $1,30 \%$ \\
\hline
\end{tabular}

Fonte: Autores.

Ao se tratar da conduta do tratamento para perda de peso, 50,95\% informaram que não realizaram nenhuma atividade física para associar com o uso dos anorexígenos, e 65,15\% das usuárias relataram mudanças nos hábitos alimentares para auxílio no tratamento.

Relacionando os medicamentos para emagrecer com os resultados no organismo das mulheres, é possível chegar no percentual de: 45,20\% que conseguiram ter redução no peso durante o uso, porém, após o término do tratamento, voltaram a engordar; 32,70\% informaram que não apresentaram resultado nem durante nem após o uso e 22,10\% apresentaram resultados satisfatórios durante e após a interrupção do uso dos medicamentos.

Os motivos que levaram as entrevistadas a recorrerem para o tratamento farmacológico podem ser analisados no Gráfico 2, cujo principal motivo foi a perda de peso. Além disso, 86,20\% das respondentes afirmaram conhecer outras mulheres que fizeram uso deste tipo de fármaco. 
Gráfico 2. Motivos para o uso dos anorexígenos

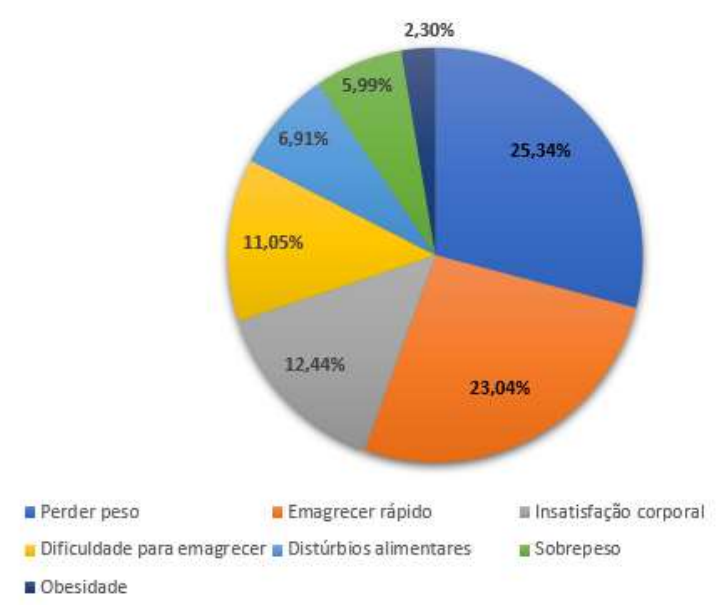

Fonte: Autores.

Quanto à orientação médica, 47,50\% das mulheres relataram não terem acompanhamento com um profissional da saúde enquanto 52,50\% foram acompanhadas. Dessas mulheres que não tiveram uma orientação, 26,21\% informaram que iniciaram a utilização dos medicamentos para emagrecer por indicação de amigos, enquanto $26,21 \%$ informaram que foi através da internet que tiveram acesso ao conhecimento do medicamento e começaram o uso. Já 21,36\% mulheres começaram o uso por conta própria, em contrapartida, 13,59\% afirmaram não se lembrar de quem a indicou, e por fim 12,63\% iniciaram a utilização por indicação de conhecidos ou familiares.

\section{Discussão}

Conforme a pesquisa realizada, a sibutramina é majoritariamente o fármaco mais utilizado para emagrecimento, onde de acordo com o Quadro 4, 41,94\% das participantes fazem uso. Ao realizar um comparativo com o estudo feito no estado de Goiás por Silva, Oliveira \& Ferreira (2012), a sibutramina também foi o fármaco mais procurado. O que difere do estudo realizado em Santa Maria no Rio Grande do Sul por um período de 6 meses por Feltrin et al. (2007), que analisava o panorama de dispensação de anorexígenos o qual acusou que os medicamentos de maior procura eram o femproporex, anfepramona e mazindol, respectivamente, devido a recorrência da prescrição. Sendo o último princípio ativo nem mesmo citado pelas mulheres respondentes neste estudo (Silva et al., 2012; Feltrin et al., 2009).

Dentre as 217 mulheres participantes da pesquisa, 22,58\% relataram o uso de mais de um anorexígeno, porém não foi especificado se houve uso concomitante de fármacos desta classe ou demais medicamentos. Sendo assim, não foi possível realizar comparações com outros estudos.

Como grande parte das participantes apresentaram efeitos colaterais $(72,25 \%)$, é de suma importância lembrar que os 5 mais citados, em ordem decrescente de porcentagem são: náuseas, dor de cabeça, diarreia, mudanças de humor e boca seca; os quais são efeitos adversos muito comuns e previstos nas bulas, conforme demonstrado nos Quadros 2 e 3, uma vez que são citados múltiplas vezes.

É importante ressaltar também que apesar de somente 3,90\% das mulheres notarem alterações na pressão arterial, este costuma ser um fator de alta relevância em estudos de anorexígenos, pois os medicamentos que possuíram o uso descontinuados e proibidos, demonstravam nos(as) pacientes patologias relacionadas ao coração. Um exemplo é a fenfluramina e seu enantiômero dexfenfluramina, que foram descontinuados devido às doenças valvares do coração e hipertensão pulmonar, efeito o qual não foi detectado durante a fase de pesquisa clínica (Waldron, 2017). Com base em 
relatórios de eletrocardiogramas de pessoas que fizeram o uso deste medicamento, foi confirmado que é um potente fator das valvulopatias apresentadas pelos indivíduos (Hopkins \& Polukoff, 2003).

Além disso, a sibutramina que foi o fármaco de maior destaque deste estudo, passou por uma pesquisa denominada SCOUT (Sibutramine Cardiovascular Outcome Trial) entre os anos de 2003 e 2009 em 16 países (James et al., 2010), que avaliava sua eficácia e segurança em pessoas com sobrepeso e obesas (Campos et al., 2014) de IMC entre 27 e $45 \mathrm{~kg} / \mathrm{m}^{2} \mathrm{com}$ comorbidades em doenças cardiovasculares ou ainda associadas de diabetes mellitus (James, 2005). Ainda assim, é importante ressaltar que a perda de peso é um processo que inclui atividade física diária e alimentação balanceada rica em frutas, verduras e grãos integrais, e restrita a açúcares, sal e gorduras (Oliveira, Carvalho, Gonçalves \& Vanzin, 2014), e que a medicação somente faz parte do processo de emagrecimento caso a alimentação e a prática de exercícios mostrem-se ineficazes em pacientes obesos (Moreira \& Alves, 2015).

Apesar de no fim do SCOUT as comorbidades não terem aumentado o índice de mortalidade dos pacientes (Cheung, 2011), foi concluído que a sibutramina deve ser apenas prescrita para pacientes sem histórico de doenças cardiovasculares (James et al., 2010), o que reforça os efeitos adversos demonstrados no Quadro 2. O uso deste fármaco deve ser acompanhado, pois $47,50 \%$ das mulheres que responderam ao formulário não tiveram acompanhamento profissional e iniciaram o uso de anorexígenos por conta própria, pela influência da internet ou por indicação de amigos, familiares e até mesmo conhecidos.

Os anorexígenos são medicamentos que somente são recomendados caso o tratamento através de dietas prescritas e atividades físicas não estejam surtindo efeito. Além disso, a pessoa precisa ser classificada obesa conforme dito na introdução e, de acordo com os resultados da pesquisa, apenas 2,30\% das mulheres são obesas. A pesquisa também demonstrou um baixo índice de adesão as atividades físicas com apenas 49,05\%, e apesar da adesão da mudança de alimentação ter sido maior (65,15\%), ambos os parâmetros são baixos, pois o tratamento farmacológico é apenas um suporte e não uma medida inicial, sendo assim, diferente do que $23,04 \%$ das mulheres pensam, pois iniciaram o uso destes medicamentos como uma forma de emagrecer rápido e sem esforço. Este fator é ainda mais evidenciado em Santa Maria que relatou a venda de anorexígenos mais evidente no verão sendo 93,35\% adquiridos por mulheres (Feltrin et al., 2009).

O emagrecimento saudável é um processo constante de reeducar hábitos alimentares, aliado a prática de atividades físicas diárias, sendo o tratamento farmacológico apenas uma medida auxiliar, pois a perda de peso através de fármacos sem a associação de exercícios pode acarretar a perda de massa muscular, ao invés da perda desejada de tecido adiposo (Massuia, Bruno \& Silva, 2008). Portanto é esperado que ao interromper o uso haja a recuperação de parte do peso; e foi o que ocorreu com 45,20\% das mulheres; na qual pode ter ocorrido por não terem mantido as mudanças de hábitos realizadas durante o uso do medicamento, diferente das $22,10 \%$ as quais o resultado se manteve.

\section{Considerações Finais}

Através deste estudo foi possível concluir que o tratamento farmacológico para emagrecimento não vem sendo aplicado da maneira correta, pois os anorexígenos são uma medida de auxílio para perda de peso em casos de obesidade, os quais a implementação da atividade física e modificações na alimentação não obtiveram os resultados desejados. Isto evidencia que os riscos e efeitos colaterais destes fármacos não foram devidamente avaliados por mulheres que foram em busca de um emagrecimento rápido e sem acompanhamento de profissionais da saúde.

Visto que estes medicamentos possuem uma lista de efeitos adversos, o que inclui tolerância e dependência; a sibutramina foi o fármaco mais citado pelas participantes da pesquisa e seu uso a longo prazo pode causar diversas patologias, tais como doenças cardiovasculares. 
Research, Society and Development, v. 10, n. 13, e62101321005, 2021

(CC BY 4.0) | ISSN 2525-3409 | DOI: http://dx.doi.org/10.33448/rsd-v10i13.21005

\section{Referências}

Ades, L. \& Kerbauy, R. R. (2002). Obesidade: realidades e indagações. Psicologia USP, 13 (1). https://doi.org/10.1590/S0103-65642002000100010.

Amaral, O. \& Pereira, C. (2016). Obesidade da genética ao ambiente. Millenium, 34 (13), 311-322. https://revistas.rcaap.pt/millenium/article/view/8374.

Balthar, R. O.; Maciel, A. P. O. A. \& Ferreira, C. C. D. (2021). Benefits of the flour of the yellow passion fruit (Passiflora Edulis F. Flavicarpa Deg.) in the treatment of Diabetes mellitus type 2: A narrative review. Research, Society and Development, 10 (9). https://doi.org/10.33448/rsd-v10i9.18404.

Barros, Y. I. B. (2019). Imagem corporal e a influência da mídia na construção do corpo feminino. Monografia, Universidade Católica de Salvador. http://ri.ucsal.br:8080/jspui/handle/prefix/945.

Campos, L. S.; Oliveira, L. A.; Silva, P. K. P. \& Paiva, A. M. R. (2014). Estudo dos efeitos da sibutramina. Revista Uningá Review, 20 (3), $50-53$. http://34.233.57.254/index.php/uningareviews/article/view/1601.

Claudino, A. M. \& Zanella, M. T. (2004). Guia de Medicina Ambulatorial e Hospitalar UNIFESP/Escola Paulista de Medicina: Transtornos Alimentares e Obesidade. $1^{\text {a }}$ edição. Editora Manole.

Conte, S. C. \& De Campos, S. B. (2015). Perspectivas de perda de peso com o uso da liraglutida: revisão literária. Brazilian Journal of Surgery and Clinical Research, 10 (1), 84-90. https://www.mastereditora.com.br/periodico/20141130_215615.pdf.

Cheung, B. M. Y. (2011). Drug treatment for obesity in the post-sibutramine era. Drug-Safety, 34, 641-650. https://doi.org/10.2165/11592040-00000000000000 .

Duarte, A. P. N. B.; Govato, T. C. P.; Carvalho, R. G.; Pontes-Júnior, L. C. B.; Rodrigues, C. L.; Santos, G. M. P.; Nicolau, L. A. D.; Ferraz, R. R. N. \& Menezes-Rodrigues, F. S. (2020). Uso de anfepramona, femproporex, mazindol e sibutramina no tratamento de pacientes com sobrepeso ou obesidade: análise farmacológica e clínica. International Journal of Health Management Review, 6 (2). https://ijhmreview.org/ijhmreview/article/view/210/142.

Dumith, S. C.; Menezes, A. M. B.; Bielemann, R. M.; Petresco, S.; Silva, I. C. M.; Linhares, R. S.; Amorim, T. C.; Duarte, D. V.; Araújo, C. L. P. \& Santos, J. V. (2012). Insatisfação corporal em adolescentes: um estudo de base populacional. Ciência \& Saúde Coletiva, 17 (9), $2499-2505$.

https://www.scielosp.org/article/csc/2012.v17n9/2499-2505/.

Feltrin, A. C.; Zordan, G.; Wagner, F.; Schmitt, G. C.; Boligon, A. A.; Delamolle, N.; Athayde, M. L. \& Vaucher, L. C. (2009). Medicamentos anorexígenos panorama da dispensação em farmácias comerciais de Santa Maria (RS). Revista do Centro de Ciências da Saúde, 35 (1), $46-51$. https://periodicos.ufsm.br/revistasaude/article/view/6529/3990.

Ferrari, G. S. L. \& Ferrari, C. K. B. (2017) Body image insatisfaction: a gender approach among Brazilian adolescents. Archiv Euromedica, 7 (1), 14-19. http://journal-archiveuromedica.eu/euromedica_01_2017/archiv_euromedica_01_2017_maket_08_08_2017_01_83_NEW.pdf.

Ferreira, R. A. B. \& Benicio, M. H. D. A. (2015). Obesidade em mulheres brasileiras: associação com paridade e nível socioeconômico. Revista Panamericana de Salud Pública, 37 (4/5), 337-342. https://www.scielosp.org/article/rpsp/2015.v37n4-5/337-342/.

Fortes, R. C.; Guimarães, N. G.; Haack, A.; Torres, A. A. L. \& Carvalho, K. M. B. (2006). Orlistat e sibutramina: bons coadjuvantes para perda e manutenção de peso? Revista Brasileira de Nutrição Clínica, 21 (3), 244-251. http://www.luzimarteixeira.com.br/wp-content/uploads/2010/10/inibidores-de-apetite.pdf.

Franke, S. I. R.; Burgos, M. S.; Reuter, C. P.; Burgos, L. T.; Pohl, H. H.; Pauli, L. T. S.; Horta, J. A.; Reckziegel, M. B.; Prá, D. \& Camargo, M. (2010). Uma análise entre índices pressóricos, obesidade e capacidade cardiorrespiratória em escolares. Arquivos Brasileiros de Cardiologia, 94 (6). https://doi.org/10.1590/S0066-782X2010005000046.

Gil, A. C. (2017). Como elaborar projetos de pesquisa. $6^{\mathrm{a}}$ edição. Atlas.

Hopkins, P. N. \& Polukoff, G. I. (2003). Risk of valvular heart disease associated with use of fenfluramine. BMC Cardiovasc Disord, 3 (5). https://doi.org/10.1186/1471-2261-3-5.

James, W. P. T. (2005). The SCOUT study: risk-benefit profile of sibutramine in overweight high-risk cardiovascular patients. European Heart Journal Supplements, 7 (1), 44-48. https://doi.org/10.1093/eurheartj/sui086.

James, W. P. T.; Caterson, I. D.; Coutinho, W.; Finer, N.; Van Gaal, L. F.; Maggioni, A. P.; Torp-Pedersen, C.; Sharma, A. M.; Shepherd, G. M.; Rode, R. A. \& Renz, C. L. (2010). Effect of sibutramine on cardiovascular outcomes in overweight and obese subjects. The New England Journal of Medicine, 363, 905917. Doi: 10.1056/NEJMoa1003114.

Kaoutar, K.; Hilali, M. K. \& Loukid, M. (2019). Insatisfaction corporelle, et attitude à l'égard de poids chez l'adolescent scolarisé au Maroc. Antropo, 42, 4553. http://www.didac.ehu.es/antropo/42/42-04/Kaoutar.pdf.

Marcon, C.; Silva, L. A. M.; Moraes, C. M. B.; Martins, J. S. \& Carpes, A. D. (2012). Uso de anfetaminas e substâncias relacionadas na sociedade contemporânea. Disciplinarum Scientia, 13 (2), 247-263. https://periodicos.ufn.edu.br/index.php/disciplinarumS/article/view/1018/963.

Marconi, M. A. \& Lakatos, E. M. (2017). Metodologia do trabalho científico. $8^{\text {a }}$ edição. Atlas.

Massuia, G. R.; Bruno, T. I. B. \& Silva, V. L. (2008). Regime de emagrecimento X utilização de drogas. Revista Científica UNIFAE, 2 (1), 5-9.

https://www.fae.br/2009/PensamentoPlural/Todas/artigo21_regimedeemagrecimentoxutilizacao\%20de\%20drogas.pdf.

Mendes, C. F. O. (2018). Assistência farmacêutica na obesidade: uma nova análise. Monografia, Universidade Federal de Ouro Preto. https://monografias.ufop.br/handle/35400000/1057. 
Research, Society and Development, v. 10, n. 13, e62101321005, 2021

(CC BY 4.0) | ISSN 2525-3409 | DOI: http://dx.doi.org/10.33448/rsd-v10i13.21005

Mendonça, C. P. \& Anjos, L. A. (2004). Aspectos das práticas alimentares e da atividade física como determinantes do crescimento do sobrepeso/obesidade no Brasil. Cadernos de Saúde Pública, 20 (3), 698-709. https://doi.org/10.1590/S0102-311X2004000300006.

Moreira, F. \& Alves, A. A. (2015). Utilização de anfetaminas como anorexígenos relacionados à obesidade. Revista Científica da FHO, 3 (1), 84-91. http://www.uniararas.br/revistacientifica/_documentos/art.9-029-2015.pdf.

Muakad, I. B. (2013). Anfetaminas e drogas derivadas. Revista da Faculdade de Direito, 108, 545-572. https://www.revistas.usp.br/rfdusp/article/view/67996.

Naccarato, M. C. \& Lago, E. M. O. (2014). Uso dos anorexígenos anfepramona e sibutramina: benefício ou prejuízo à saúde? Revista Saúde, 8 (1/2) 66-72. http://revistas.ung.br/index.php/saude/article/view/834.

Nappo, S. A.; Tabach, R.; Noto, A. R.; Galduróz, J. C. F. \& Carlini, E. A. (2002). Use of anorectic amphetamine-like drugs by Brazilian women. Eating Behaviors, 3 (2), 153-165. https://doi.org/10.1016/S1471-0153(01)00054-X.

Nateghi, S. \& Effatpanah, H. (2020). An epidemic amphetamine problem: commentary on treatment of amphetamine abuse/use disorder. DARU Journal of Pharmaceutical Sciences, 28 (1), 417-418. https://dx.doi.org/10.1007\%2Fs40199-019-00313-z.

Oliveira, J. S. B.; Carvalho, K. D.; Gonçalves, R. M. B. \& Vanzin, S. D. B. (2014). Aspectos relevantes do uso indiscriminado de fármacos para perda de peso. Revista FUNEC Científica - Nutrição, 1 (2). https://seer.unifunec.edu.br/index.php/rfcn/article/view/1121.

Orsi, J. V. A.; Nahas, F. X.; Gomes, H. C.; Andrade, C. H. V.; Veiga, D. F.; Novo, N. F. \& Ferreira, L. M. (2008). Impacto da obesidade na capacidade funcional de mulheres. Revista da Associação Médica Brasileira, 54 (2). https://doi.org/10.1590/S0104-42302008000200010.

Pinheiro, A. R. O.; Freitas, S. F. T. \& Corso, A. C. T. (2004). Uma abordagem epidemiológica da obesidade. Revista de Nutrição, 17 (4). https://doi.org/10.1590/S1415-52732004000400012.

Rang \& Dale. (2016). Farmacologia. $8^{\mathrm{a}}$ edição. GEN Guanabara Koogan.

Robledo, P. (2008). Las anfetaminas. Transtornos Adictivos, 10 (3), 166-174. https://doi.org/10.1016/S1575-0973(08)76363-3.

Santos, K. P.; Silva, G. E. \& Modesto, K. R. (2019). Perigo dos medicamentos para emagrecer. Revista de Iniciação Científica e Extensão, 2 (1), $37-45$. https://revistasfacesa.senaaires.com.br/index.php/iniciacao-cientifica/article/view/140.

Silva, J. R.; Oliveira, E. N. F. \& Ferreira, A. G. (2012). Avaliação do consumo de anorexígenos derivados de anfetaminas em cidades de Goiás. Ensaios e Ciência, 16 (3), 9-19. https://doi.org/10.17921/1415-6938.2012v16n3p\%25p

Skrsypcsak, C. \& Locatelli, C. (2013). Efeitos da liraglutida sobre a glicemia e a obesidade: uma revisão bibliográfica. Vita et Sanitas, 7 (1), 51-64. http://fug.edu.br/revistas/index.php/VitaetSanitas/article/view/41.

Soares, R. (2019). O percurso das drogas no império da anfetamina. História, Ciências, Saúde-Manguinhos, 26 (2). https://doi.org/10.1590/S010459702019000200023.

Soyka, M. (2017). Treatment of benzodiazepine dependence. The New England Journal of Medicine, 376 (12), 1147-1157. Doi: 10.1056/NEJMra1611832.

Sousa, D. V. (2015). Anfetaminas: efeitos, mecanismos de ação, usos clínicos e de abuso. Monografia, Universidade Federal de Campina Grande. http://dspace.sti.ufcg.edu.br:8080/jspui/handle/riufcg/9059.

Tavares, T. B.; Nunes, S. M. \& Santos, M. O. (2010). Obesidade e qualidade de vida: revisão de literatura. Revista Médica de Minas Gerais, 20 (3), 359-366. http://rmmg.org/artigo/detalhes/371.

Utrilla, P. (2000). Aspectos farmacológicos de las anfetaminas. Ars Pharm, 41 (1), 67-77. https://revistaseug.ugr.es/index.php/ars/article/view/5734.

Wadden, T. A \& Foster, G. D. (2000). Behavioral treatment of obesity. Medical Clinics of North America, 84 (1), 441-461. https://doi.org/10.1016/S00257125(05)70230-3.

Waldron, K. (2017). Managing risk to the patient: recording quality risk management for the pharmaceutical and biopharmaceutical industries. Doctoral thesis, DIT. https://doi.org/10.21427/D7G165. 